\title{
Hemodynamic Stroke in Simultaneous Cardio Cerebral Infarction: A New Term for Cardiologist
}

\author{
Mochamad Yusuf $^{1 *(D)}$, Ivan Satria Pratama ${ }^{1}$, Ruth Gunadi ${ }^{1}$, Achmad Firdaus Sani ${ }^{2}$ (D) \\ ${ }^{1}$ Department of Cardiology and Vascular Medicine, Universitas Airlangga, Dr. Soetomo Hospital, Surabaya, East Java, \\ Indonesia; ${ }^{2}$ Department of Neurology, Universitas Airlangga, Dr. Soetomo Hospital, Surabaya, East Java, Indonesia
}

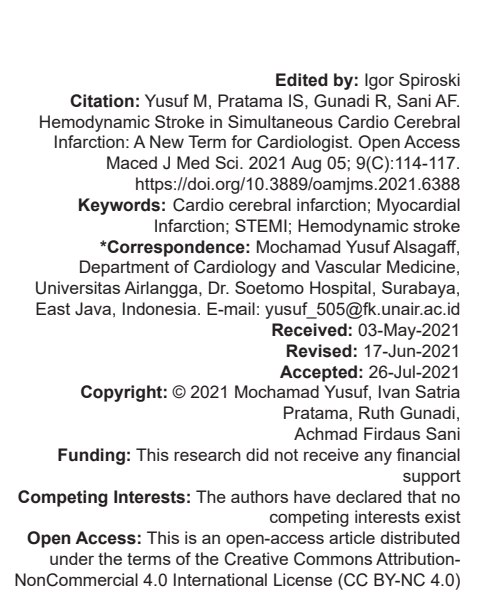

Abstract

BACKGROUND: Incidence of cardio cerebral infarction is exceptionally rare, with an incidence rate as low as $0.009 \%$. Hemodynamic compromise in patients with acute myocardial infarction may result in the cerebral blood flow reduction to water-shed areas of the brain that cause hemodynamic stroke.

CASE REPORT: We report an uncommon case of simultaneous cardio-cerebral infarction, due to Inferior right ventricle ST Segment Elevation Myocardial Infarction (STEMI) with acute ischemic stroke. Due to the rarity of this condition, there were no recommended therapeutics strategy. Mechanical reperfusion with $\mathrm{PCl}$ procedure may be a superior choice in the settings of acute cardio-cerebral infarction for restoring hemodynamic stability.

CONCLUSION: Therefore, $\mathrm{PCl}$ should be reasonable approach in treating patients with simultaneous cardio cerebral infarction.

\section{Introduction}

Cardiovascular events persist to be the leading cause of death worldwide and continue to grow in number. Mortality is predisposed by numerous factors such as advanced age, Killip class, left ventricular ejection fraction (LVEF), and complications following myocardial infarction (MI), for example, acute stroke [1], [2]. Recent studies have revealed that ischemic stroke after an acute $\mathrm{Ml}$ is coupled with poorer outcomes compared to patients without cerebrovascular complications. The coinciding presentation of these conditions is acknowledged as cardio-cerebral infarction, which is known to be remarkably uncommon but precedes a life-threatening state. Decisive and precise diagnosis followed by immediate management should be achieved to minimize morbidity and mortality risks [3], [4].

\section{Case Report}

A 56-year-old male, with a history of type 2 diabetes and hypertension, presented to emergency ward with severe chest pain, on-going for $2 \mathrm{~h}$ before admission. After evaluation, the patient was diagnosed with inferior and right ventricle ST-segment elevation MI (STEMI) and total atrioventricular (AV) block. Since he presented himself to a non-percutaneous coronary intervention (PCI) capable hospital, he was prearranged for a thrombolytic procedure. However, before the procedure, he suddenly experienced hemiparesis, dysarthria, and gradual loss of consciousness. Thrombolytics procedure was postponed and a non-contrast brain computed tomography (CT) scan was performed, which revealed signs of acute ischemic stroke. Due to the risk of hemorrhagic stroke transformation, coronary reperfusion via thrombolytics was finally canceled. Hence, he was then referred to tertiary hospital for PCl.

Upon arrival, the patient was soporous with a Glasgow Coma Scale of 8 (E2 V1 M5) with his National Institutes of Health Stroke Scale (NIHSS) was 17. His blood pressure was 140/100 mm Hg, his pulse was 55 bpm. Electrocardiogram (ECG) revealed a high degree AV block with a 2:1 conduction ratio and ST-segment elevation in the inferior and posterior lead, with increased Troponin I level (1.910 $\mathrm{ng} / \mathrm{ml})$ and HbA1C $(10,3 \mathrm{mg} / \mathrm{dL})$. Echocardiography showed a reduction of LVEF and hypokinetic segments in inferior, inferoseptal, and posterior left ventricle (LV) segments without sign 
of intracardiac thrombus. A second Brain CT scan was performed and revealed no signs of hemorrhage, but there was cerebral infarction in the left centrum semiovale, left corona radiata, lacunar cerebral infarction in the right genu internal capsule (Figure 1). Afterward, the patient was immediately transferred to the cardiac catheterization lab for primary $\mathrm{PCl}$.

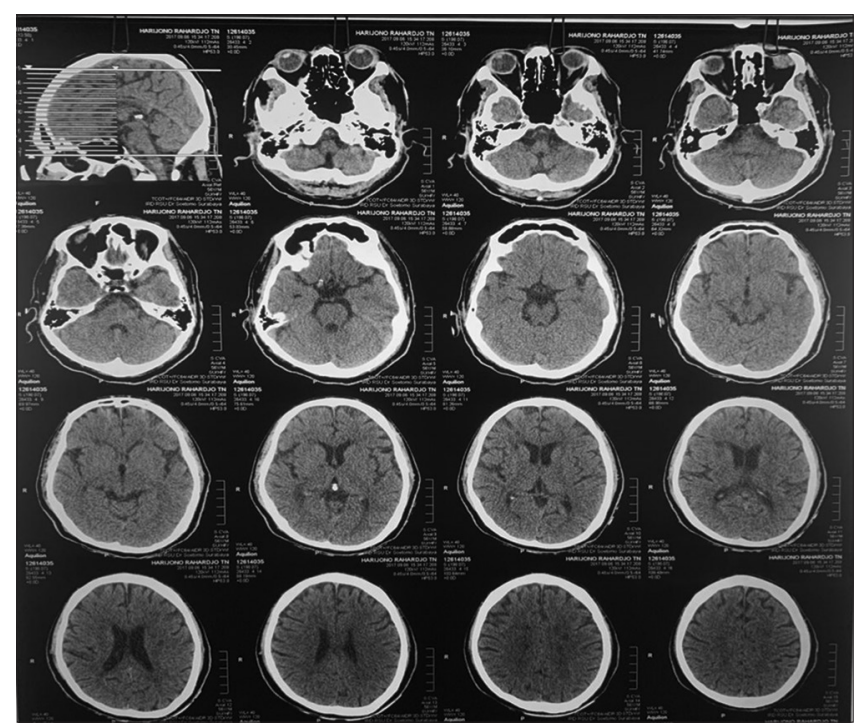

Figure 1: Computed tomography brain imaging showing infarction in the border zone area

Diagnostic coronary angiography revealed triple vessel disease, with critical stenosis at the ostial and total occlusion at mid-right coronary artery (RCA) (Figure 2).
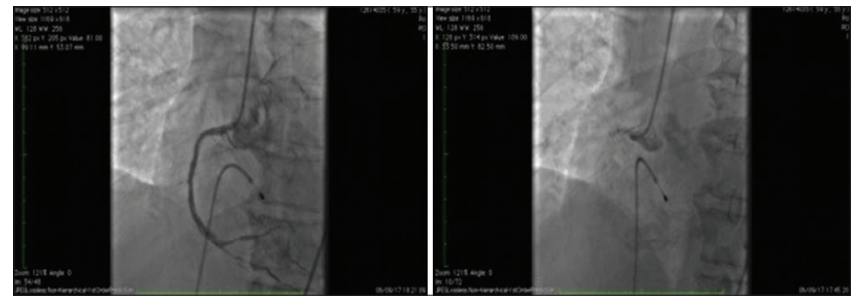

Figure 2: Pre percutaneous coronary intervention procedure on the right coronary artery (upper) and after the procedure (bottom)

Grade 2 collateral was existent from the left circumflex which was also diffusely diseased. There was a long significant $80 \%$ stenosis at proximal left anterior descending (Figure 3).
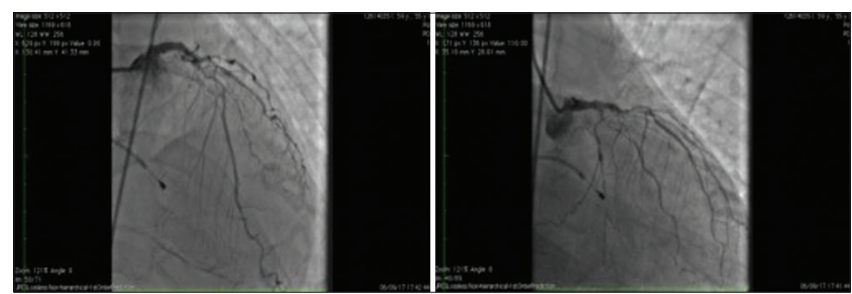

Figure 3: Diffuse disease in left anterior descending and left circumflex

The first procedure in the catheterization lab was temporary transvenous pacemaker insertion, with an initial setting of $70 \mathrm{ppm}, 2 \mathrm{~V}$ output, and $5 \mathrm{mV}$ sense. Loading dose of antiplatelets (Clopidogrel $600 \mathrm{mg}$ and Aspirin $300 \mathrm{mg}$ ) were given through nasogastric tube and Heparin 5000 IU was given intravenously.
The lesion was crossed using guidewire run-through hypercoat, and pre-dilation was carried out with a $2.0 \times 20 \mathrm{~mm}$ balloon (6 atm) at proximal RCA, $1.5 \mathrm{~mm} \times 15 \mathrm{~mm}$ balloon (16 atm) at proximal to mid-RCA, and $2.75 \mathrm{~mm} \times 20 \mathrm{~mm}$ balloon (8 atm) at proximal to mid-RCA. Thrombus aspiration was performed, revealing a red thrombus. This was followed by the placement of two sirolimus-eluting stents in RCA, $3.5 \mathrm{~mm} \times 20 \mathrm{~mm}$ and $3.5 \mathrm{~mm} \times 28 \mathrm{~mm}$, overlapped, inflated to $12 \mathrm{~atm}$, and further post dilated with a noncompliant balloon at 18 atm. Post- $\mathrm{PCl}$ angiography showed Thrombolysis in MI 3 flow in the RCA.

Post-PCl procedure, his rhythm subsequently converted to a $1^{\text {st }}$ degree AV Block, henceforth his temporary pacemaker was later removed. The patient regained consciousness in $<24 \mathrm{~h}$ post-procedure, but still had left hemiparesis, which gradually improved over time. At 1-year follow-up, he had no residual paresis and no symptoms of angina and dyspnea.

\section{Discussion}

Acute $\mathrm{MI}$ and acute ischemic stroke are medical emergencies that require timely diagnosis and management. It has been shown that acute $\mathrm{Ml}$ increases the risk of acute ischemic stroke and vice versa. Coinciding presentation of these conditions has seldom been reported and is also acknowledged as acute cardio-cerebral infarction, with an incidence estimated to be as low as $0.009 \%$ [1]. Cardio-cerebral infarction can further be divided into two classifications, first is "synchronous cardio-cerebral infarction" which is a simultaneous infarction in the cerebral and coronary vascular territories, and "metachronous cardio cerebral infarction" which is one event preceding the other [1], [2]. The reported prevalence and incidence have mostly been for the metachronous presentation of cardio-cerebral infarction. The incidence of simultaneous cardio cerebral infarction is currently unknown due to the rarity of this co-occurrence [3]. Simultaneous cardio cerebral infarction can be diagnosed by the presence of simultaneous acute onset of a focal neurological deficit - indicating acute stroke, and chest pain or evidence of $\mathrm{MI}$ such as changes of the ECG and elevation of cardiac enzymes.

The pathophysiology leading to simultaneous cardio-cerebral infarction can be multi-factorial, with proposed mechanism as follows: (i) conditions resulting in concurrent cerebral coronary infarction, (ii) cardiac diseases leading to cerebral infarction, and (iii) brainheart axis dysregulation [4], [5]. Other conditions that may contribute include: Atrial fibrillation, aortic dissection, and pre-existing cardiac thrombus [6]

Epidemiologic studies have proven that patients who have an acute $\mathrm{MI}$ have an increased risk 
of ischemic stroke. A large MI predisposes the formation of a LV mural thrombus, and befits a potential source of embolic events [7]. Another mechanism that may cause acute ischemic stroke is brain hypoperfusion resulting from acute $\mathrm{Ml}$. The occurrence of a sudden hemodynamic compromise in patients presenting with acute $\mathrm{Ml}$ may result in the reduction of cerebral blood flow to water-shed areas of the brain, especially if there is a failure of blood pressure autoregulatory mechanisms. Stroke in this condition is also known as a hemodynamic stroke [8].

The term hemodynamic stroke has traditionally been used for ischemic stroke caused by hypoperfusion and not by emboli or by local vasculopathy, where hypoperfusion can result from either regional or systemic origin. The feature that distinguishes hemodynamic stroke from other subtypes of stroke is that blood flow toward the brain is exceedingly low and results in ischemia in an area of the brain [8].

Diagnosis of a hemodynamic stroke may be difficult and is often made only after excluding a possible embolic source. Hemodynamic stroke has a distinctive pattern in brain imaging, the so-called "rosary like" pattern in the watershed area or also known as border zone (or watershed) infarct [9], [10]. Cerebral watershed infarct (WI), involves the junction of the distal fields of non-anastomosing arterial systems. There are two types of WI: cortical WI, occurring at the junction between cortical territories of the anterior, middle, and posterior cerebral arteries; and internal WI, occurring in the white matter between the deep and superficial perfusion systems of the middle cerebral artery. Pathogenesis of WI remains unthoroughly understood, and diagnosis can be challenging. In an acute phase setting, diffusion-weighted brain magnetic resonance imaging allows a clearer diagnosis than CT scan [11].

Simultaneous cardio-cerebral infarction requires early diagnosis and treatment since the myocardial cells and neurons undergo immediate necrosis. The main therapeutic strategy for this condition is to reduce infarct size by providing prompt reperfusion to the occluded artery [4]. However, due to the rarity of concurrent cardio-cerebral infarction, the recommendation for optimal reperfusion strategy in this scenario is not well established. Furthermore, a consensus among cardiologists and neurologists has not been perceived, producing challenges and dilemmas for both parties. A delayed intervention of one infarcted territory for the other may result in permanent irreversible morbidity or disability and even death. In addition to the dilemma of the sequence of management, the agents of management for each territory may complicate the extent of the other infarcted territory [3].

Intravenous thrombolytic therapy with tissue plasminogen activator (tPA) is a standard treatment for acute ischemic stroke for patients who arrived within $4.5 \mathrm{~h}$ after onset unless contraindicated and thrombolytic therapy within $12 \mathrm{~h}$ after onset, is an alternative treatment for STEMI in the setting of the non-PCl capable hospital [12], [13]. Although fibrinolytic therapy using intravenous tPA can be used in both acute ischemic stroke and acute STEMI, different dose requirements and timing of the fibrinolytic therapy after onset hinder the use of TPA as a definitive treatment for both conditions. Moreover, the higher dose and longer infusion time of tPA treatment for STEMI when compared with a standard dose for acute ischemic stroke may increase the risk of hemorrhagic transformation among patients with simultaneous acute ischemic stroke and MI [2]. A few cases of treatment success have been reported previously, using administered intravenous tPA for stroke treatment dose $(0.9 \mathrm{mg} / \mathrm{kg}$ over $60 \mathrm{~min})$, although there is an improvement in neurological status, the cardiac result is unknown [4].

The American Heart Association and American Stroke Association in 2018 recommended that in the setting of hyper-acute simultaneous cardio-cerebral infarction, treatment with intravenous alteplase at the dose used for cerebral ischemia followed by $\mathrm{PCl}$ is reasonable [1]. However, different dose requirements and timing of fibrinolytic treatment often hinder the use of alteplase as a definitive treatment for both conditions. Furthermore, suspending reperfusion therapy can lead to both hemodynamic instability and cardiac complications.

There are several anecdotal reports of simultaneous cardio-cerebral infarction with varying approaches to management. Omar Obaid et al. reported a 48-year-old patient presenting with inferoposterior and right ventricular transmural acute myocardial infarction and a massive cerebral infarction immediately after admission. The patient was treated conservatively with antiplatelet and anticoagulants but deceased the second day [2]. Another case of simultaneous cardio cerebral infarction, reported a 44-year-old male presenting with acute ischemic stroke and an NIHSS score of 11, and was found to have an inferior acute myocardial infarction with a 2:1 atrioventricular block. The patient was treated with an intravenous tissue plasminogen activator (0.9 $\mathrm{mg} / \mathrm{kg}$ over 1 hour, with a total dose of $80 \mathrm{mg}$ ). The patient, however, had recurrent episodes of malignant refractory arrhythmias including ventricular fibrillation requiring multiple resuscitation efforts [3].

These case reports highlighted the need to individualize treatment in patients presenting with simultaneous cardio-cerebral infarction. The ideal management of simultaneous cardio-cerebral infarction is a treatment strategy that benefits both vascular territories. An important deciding factor in the approach to management is the presentation of STEMI. According to the European Society of Cardiology guidelines for STEMI, $\mathrm{PCl}$ is recommended/indicated for STEMI patients within 12 after onset, or more if there were hemodynamic instability, arrhythmia, or ongoing chest 
pain [12], [14]. We would like to emphasize $\mathrm{PCl}$ as an alternative and precedence treatment for this condition, as presented in our case where $\mathrm{PCl}$ showed satisfactory results in both cardiac and neurological status.

Due to the paucity of cardio-cerebral infarction, recommendations for ideal reperfusion strategy in this scenario are still deficient. In case of suspected cardiocerebral infraction, after an initial evaluation, noncontrast CT brain, CT angiogram, and ECG should be done to confirm diagnosis. Reperfusion strategy using tPA may be selected in stable hemodynamic patients. Whereas, emergency $\mathrm{PCl}$ is required for patients who have unstable hemodynamic status or STEMI [2]. A reasonable approach to the acute management of simultaneous cardio-cerebral infarction is a combined treatment of both vascular territories with the administration of intravenous tPA at $0.9 \mathrm{mg} / \mathrm{kg}$ infused for $60 \mathrm{~min}$, with $10 \%$ of the total dose administered as an initial intravenous bolus for $1 \mathrm{~min}$, followed by $\mathrm{PCl}[2],[3]$.

\section{Conclusion}

The concurrent presentation of acute $\mathrm{MI}$ and acute ischemic stroke (also referred as cardio-cerebral infarction) is uncommon but proves to be challenging and devastating for both physician and patient. Stroke in this situation can also be described as hemodynamic stroke, where sudden hemodynamic compromise in patients with acute $\mathrm{Ml}$ may result in the cerebral blood flow reduction to water-shed areas of the brain. Considering the insufficient clinical trials and studies, treatment options should be individualized per case. Mutual therapeutic strategies and efficient team-work among neurologists and cardiologists are required for ideal management for such exceptional cases. We believe that invasive treatment using $\mathrm{PCl}$ should be reasonable. Further study is needed to determine the ideal treatment for this condition.

\section{References}

1. Dutta $M$, Das $P$, Steinhubl R. Incidence and prevention of ischemic stroke following myocardial infarction: Review of current literature. Cerebrovasc Dis. 2006;22(5-6):331-9. https:// doi.org/10.1159/000094847

PMid:16888371

2. Obaid O, Brancheau D. Simultaneous acute anterior ST-elevation myocardial infarction and acute ischemic stroke of left middle cerebral artery : A case report. Am J Case Rep. 2019;20:776-9. https://doi.org/10.12659/ajcr.916114

\section{PMid:31154453}

3. Kijpaisalratana N, Chutinet A, Suwanwela NC. Hyperacute simultaneous cardiocerebral infarction: Rescuing the brain or the heart first? Front Neurol. 2017;8:1-7. https://doi.org/10.3389/ fneur.2017.00664

PMid:29270151

4. Akinseye OA, Shahreyar M, Heckle MR, Khouzam RN. Simultaneous acute cardio-cerebral infarction: Is there a consensus for management? Ann Transl Med. 2018;6(1):7. https://doi.org/10.21037/atm.2017.11.06

PMid:29404353

5. Gungoren F, Besli F, Tanriverdi Z, Kocaturk O. Optimal treatment modality for coexisting acute myocardial infarction and ischemic stroke. Am J Emerg Med. 2019;37(4):795.e1-4. https://doi. org/10.1016/j.ajem.2018.12.060

PMid:30612780

6. Witt BJ, Ballman KV, Brown RD, Meverden RA, Jacobsen SJ, Roger VL. The incidence of stroke after myocardial infarction : A meta-analysis. Am J Med. 2006;119(4):354.e1-9. PMid:16564779

7. Hornung M, Franke J, Gafoor S, Sievert H. Cardioembolic stroke and post myocardial infarction stroke. Cardiol Clin. 2016;34(2):207-14. https://doi.org/10.1016/j.ccl.2015.12.003 PMid:27150168

8. Klijn CJ, Kappelle LJ. Hemodynamic stroke : Clinical features, prognosis, and management. Lancet Neurol. 2010;9(10):100817. https://doi.org/10.1016/s1474-4422(10)70185-x PMid:20864053

9. Bladin CF, Chambers BR. Frequency and pathogenesis of hemodynamic stroke. Stroke. 1994;25(11):2179-82. https://doi. org/10.1161/01.str.25.11.2179 PMid:7974542

10. Shi J, Meng R, Konakondla S, Ding Y, Duan Y, Wang B, et al. Cerebral watershed infarcts may be induced by hemodynamic changes in blood flow. Neurol Res. 2017;39(6):538-44. https:// doi.org/10.1080/01616412.2017.1315499 PMid:28393628

11. Juergenson I, Mazzucco S. A typical example of cerebral watershed infarct. Clin Pract. 2011;1(4):1-2. https://doi. org/10.4081/cp.2011.e114

PMid:24765355

12. Jauch EC, Saver JL, Adams HP, Bruno A, Connors JJ, Demaerschalk BM, et al. Guidelines for the early management of patients with acute ischemic stroke: A guideline for healthcare professionals from the American heart association/American stroke association. Stroke. 2013;44(3):870-947. https://doi. org/10.1161/str.0b013e318284056a PMid:29367334

13. Ibanez B, James $S$, Agewall $S$, Antunes MJ, Bucciarelli-Ducci C, Bueno H, et al. 2017 ESC Guidelines for the management of acute myocardial infarction in patients presenting with ST-segment elevation: The task force for the management of acute myocardial infarction in patients presenting with ST-segment elevation of the European society of cardiology (ESC). Eur Heart J. 2018;39(2):119-77. https://doi. org/10.5603/kp.2018.0041

PMid:28886621

14. Lanzer P, Widimsky P. Ischaemic stroke and ST-segment elevation myocardial infarction: Fast-track single-stop approach. Eur Heart J. 2015;36(35):2348-55. https://doi. org/10.1093/eurhearti/ehv217

PMid:26038589 